specific gravity as pure tin. It is known that one-half per cent. of aluminium in iron increases its specific gravity. One per cent. of antimony in lead makes an alloy heavier than lead; 2 per cent. is of the same specific gravity as lead, and higher percentages are lighter. It is very likely that these alloys will show an analogous behavior in their melting points. Alloys are best studied by making a complete correlation of as many physical properties as can be observed, and such study is not only of high theoretical interest but of immediate practical value in the arts.

\title{
MONOCHROMATIC COATINGS ON GERMAN SILVER AND PLATINUM.
}

BY DR. R. BOETTGER.

After innumerable experiments success has been achieved in producing the finest monochromatic shades on platinum and German silver by electrolytic decomposition of organic manganous salts, the metal to be treated being connected with the positive electrode. It has formerly been supposed that it was principally the form of the negative pole that influenced the nature of the Nobili figures. Although this cannot be denied altogether-for with a sharply pointed platinum wire only ring-shaped figures can be obtained-yet the rule is subject to modification with regard to certain manganous salts. With solutions of manganous hippurate, acetate or succinate, even when a very thin platinum wire serves as the negative pole, colored rings are never formed, but a monochromatic coating, on the positive electrode. This has only been observed with these special salts. It further appears that metals which form higher oxides, such as lead and manganese, are the best in solution for producing Nobili figures. In using the special manganese salts for obtaining the monochromatic coating, the shape of the negative pole appears to be immaterial; but it is advisable to secure uniformity of the coating on the positive element, that the negative element should take the form of a disk of platinum. Nothing decided can be said about the strength of the manganese solutions, as the best strength depends upon the power of the current, and must be determined by each experimenter for his own special case. The weaker the current the stronger the manganese solution must be. The color produced ehanges greatly and quickly, so that it is essential instantly to break the current when the desired color has been produced. Golden yellow, green, and purple are obtained with particular brilliancy. The metal should be removed from the bath as soon as the current is stopped, rinsed with distilled water, and carefully dried with soft blotting paper. If manganous chloride or lead acetate is used, the colors appear in rings instead of as a uniform layer and of all the colors of the rainbow, in the softest shades, the predominant hues being green, golden yellow and blue, each whole system of rings surrounded by a yellow zone.-Der Metallarbeiter. 